\title{
Error Analysis on CNC gear hobbing Machine Spindle Transmission Chain Based on SOV Theory
}

\author{
ZHAO jia-li ${ }^{1}$, LIU Yuan ${ }^{2}$, GUO Zhen ${ }^{3}$ and ZHANG Yao ${ }^{4}$ \\ School of Mechanical and Electronical Engineering, Lanzhou University of Technology, Lanzhou \\ 730050, China \\ j_I_zh@163.com,454350961@qq.com,1302789185@qq.com ,515132718@qq.com
}

\begin{abstract}
Keywords: SOV CNC gear hobbing machine State space model Error propagation
Abstract. In view of the situation that the transmission elements of YS3118 CNC gear hobbing machine are too many and complicated, leading to transmission errors which cannot guarantee the machining precision, Based on SOV theory, with CNC gear hobbing machine as example, the method of using state-space model to describe the transmission error flow delivery is proposed in this paper.First the coordinate system to describe transmission parts gear geometric characteristics is constructed, mathematical modeling of the revolute pair deviations caused by machining errors or assembly errors is fulfilled, the coordinate system transformation containing error parameters in gear drive combined with kinematics theory is described and the expressions of errors flow in transmission process using state-space model have been set up.ultimately through the calculation example, the effectiveness of this method is verified.
\end{abstract}

\section{Introduction}

Gear is an important basic parts, the precision of machines determines gear accuracy, CNC hobbing is widely used in gear machining, due to China's backward industry, a large percentage of hobbing is middle and low level, In view of the situation that the transmission elements of YS3118 CNC gear hobbing machine are too many and complicated, leading to component manufacturing and assembly errors, which makes the head and end pieces of inner drive chain do not work in terms of ideal transmission relations, resulting in transmission errors and the affecting gear machining accuracy [1], so a error analysis on YS3118 CNC hobbing spindle transmission system is of very important significance.

SOV(Stream of Variation) was initially proposed by scholars for the limitation of SPC ( Statistical Process Control), they viewed the whole manufacturing process as a dynamic system and built mathematical models, researched multi-factor coupling problems, quantificationally analyzed the entire manufacturing process to ensure and improve the quality of the final product [2]. After Assemblability concept was put forward, D.E Whitney believed matrix conversion representation between part features can be used to indicate errors propagation in assembly process, and predicted the likelihood of the assembly process success [3]. R. Mantriparagada, following the concept of the reference flow chains, regarded each work station in assembly process as a series of discrete events, used the state transition equation to express the discrete dynamic systems and the controllability of control theory was introduced to optimize the system, so assembly features were improved to reduce product errors [4-6]. Q. Z. Tian further developed state transition equation to describe the relation between multi-station fixture deviation and parts size deviation, described the assembly deviation transfer of any number of parts on arbitrary shape work station [7]. J.Hong proposed a method that mathematical expression was made on precision machine assembly measurement and adjust technology and state-space model was used to forecast machine precision [8].

Aiming at SOV of machine transmission chains, according to state space methods in control theory to describe and predict multi-technology machining and assembly deviation transfer process, this paper uses state-space equation, builds error propagation model of hobbing spindle transmission chain .By differential motion vector method as unified expression of each gear transmission part error state, the corresponding relation between the gear part tolerances model and variation sources 
mathematical expression in transmission process is built. Based on this model, the hob error vector is calculated according to gear error states .

\section{YS3118 Hobbing Structure and Spindle Drive Principle}

\section{YS3118 hobbing physical structure}

As shown in Figure 1, gear hobbing makes gear machining by means of the relative movement of hob and workpiece, which is based on linear movement and rotational movement of each kinematic pair, including $\mathrm{X}$ linear motion, $\mathrm{Z}$ linear motion, $\mathrm{A}$ swing (adjusting hob mounting angle, remaining still during processing), $\mathrm{Y}$ linear motion, $\mathrm{B}$ and $\mathrm{C}$ rotary motion, which with machine bed, hob, piece together constitute hobbing physical structure.

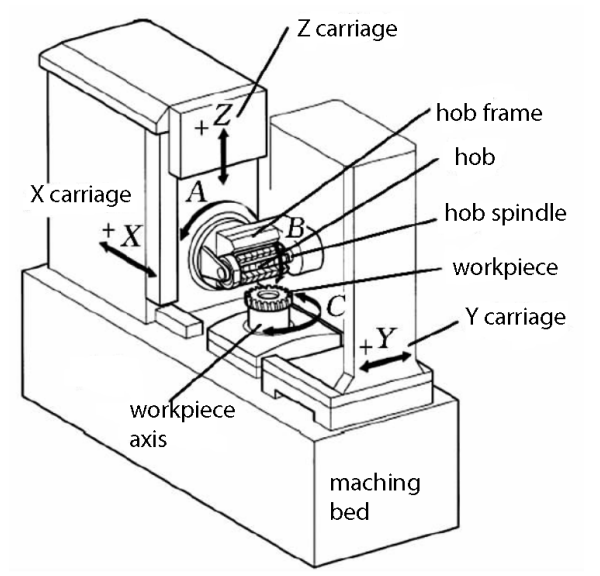

Fighre 1. hobbing physical structure diagram

\section{spindle drive principle}

Hobbing spindle drive is a complex speed control system, which consists of all levels of shifting mechanism of belt drive and gear drive shown in Figure 2, the shifting mechanism transmits motor output speed and torque through four levels of gear shaft to hob spindle. A single motor drive and split transmission mode driving a plurality of actuating mechanism are used in YS3118 CNC hobbing spindle design, The strict speed and itinerary synchronization relationship of inline transmission chain is relied on transmission components having accurate transmission ratio, and therefore gear itself manufacturing error and assembly error on the accuracy of the final hob play a major role.

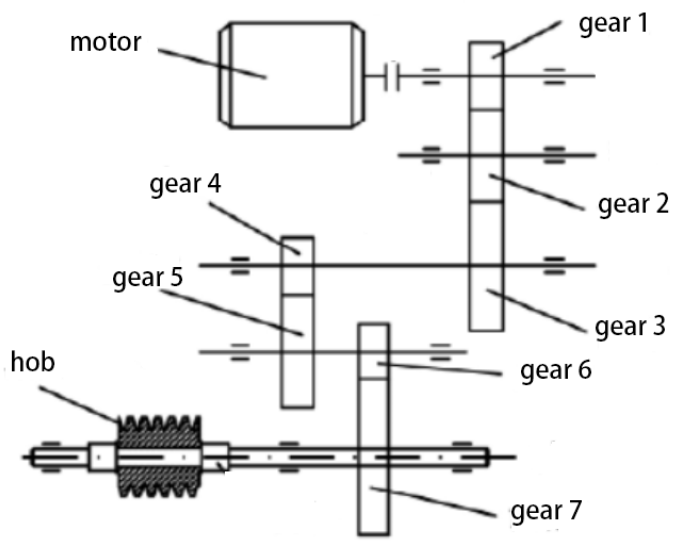

Figure 2. hobbing spindle drive system simplified model diagram 


\section{revolute pair motion error elemental analysis and transmission modeling revolute pair motion error elemental analysis}

As shown in Figure 3, under the ideal case of there is no error in gear rotary motion. But in reality, due to machining and assembly errors, the actual shaft axis offsets and tilts. When the rotating shaft rotates angle $\theta$, the error motion components of revolute pair show translational errors $\Delta x, \Delta y, \Delta z$ along three axes and tilt errors $\Delta \theta_{x}, \Delta \theta_{y}, \Delta \theta_{z}$ around three axes .

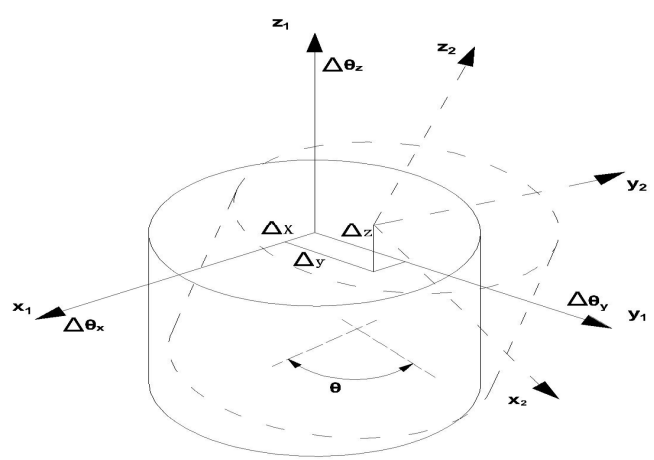

Figure 3. revolute pair actual movement schematic

\section{gear transmission error modeling}

As shown in Figure 4, the parts coordinate systems are established in the two adjacent gears $\mathrm{i}-1$ and $\mathrm{i}$, the coordinate origin is at gear geometric center ,the solid line represents ideal gear position while the dotted line indicates actual gear position when errors exist . Ideally $A_{i}$ represents the transformation matrix from the coordinate system $i-1$ to the coordinate systemi, according to kinematics theory [9]:

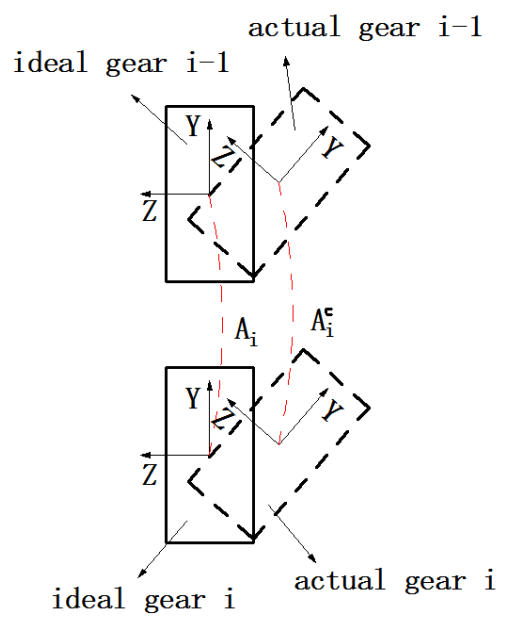

Figure 4. error propagation in gear transmission process 


$$
\left[A_{i}\right]_{\text {Rot }}=\left[A_{i}\right]_{\text {RotX }}\left[A_{i}\right]_{\text {RotY }}\left[A_{i}\right]_{\text {RotZ }}
$$

$$
\begin{array}{r}
{\left[A_{i}\right]_{\text {Transl }}=\left[\begin{array}{lllc}
1 & 0 & 0 & X_{i} \\
0 & 1 & 0 & Y_{i} \\
0 & 0 & 1 & Z_{i} \\
0 & 0 & 0 & 1
\end{array}\right]} \\
{\left[A_{i}\right]_{R o t Y}=\left[\begin{array}{cccc}
\cos \theta_{y i} & 0 & \sin \theta_{y i} & 0 \\
0 & 1 & 0 & 0 \\
-\sin \theta_{y i} & 0 & \cos \theta_{y i} & 0 \\
0 & 0 & 0 & 1
\end{array}\right]}
\end{array}
$$

$\left[A_{i}\right]_{R o t X}=\left[\begin{array}{cccc}1 & 0 & 0 & 0 \\ 0 & \cos \theta_{x i} & -\sin \theta_{x i} & 0 \\ 0 & \sin \theta_{x i} & \cos \theta_{x i} & 0 \\ 0 & 0 & 0 & 1\end{array}\right]$

$\left[A_{i}\right]_{R o t Z}=\left[\begin{array}{cccc}\cos \theta_{z i} & -\sin \theta_{z i} & 0 & 0 \\ \sin \theta_{z i} & \cos \theta_{\mathrm{zi}} & 0 & 0 \\ 0 & 0 & 1 & 0 \\ 0 & 0 & 0 & 1\end{array}\right]$

As shown in Eq.(1), $X_{i}, Y_{i}, Z_{i}$ are translation amounts along three axes and $\theta_{x i}, \theta_{y i}, \theta_{z i}$ are translation amounts around three axes from coordinate system i - 1 to i, Therefore, $A_{i}$ can be simply expressed in Eq.(2).

$A_{i}=\left[\begin{array}{cc}R_{i} & P_{i} \\ 0 & 1\end{array}\right]$

$R_{i}$ is $3 \times 3$ rotation matrix, $P_{i}$ is $3 \times 1$ translation vector

In actual case when errors exist, $A_{i}^{c}$ is expressed in Eq.(3).

$A_{i}^{c}=A_{i}+\mathrm{d} A_{i}=\left[I+\delta A_{i}\right] A_{i}$

$\delta A_{i}$ is the error transformation matrix from ideal coordinate system to actual coordinate system $i$, which can be written in Eq.(4).

$\delta A_{i}=\left[\begin{array}{cc}\delta_{i} & d_{i} \\ 0 & 0\end{array}\right]$

$\delta_{i}$ is $3 \times 3$ rotation error matrix, $P_{i}$ is $3 \times 1$ translation error vector.

According to differential transformation principle, $d A_{i}$ can be expressed in Eq.(5)

$\mathrm{d} A_{i}=\left[\frac{\partial}{\partial X_{i}} A_{i}\right] \mathrm{d} X_{i}+\left[\frac{\partial}{\partial Y_{i}} A_{i}\right] \mathrm{d} Y_{i}+\left[\frac{\partial}{\partial Z_{i}} A_{i}\right] \mathrm{d} Z_{i}$
$+\left[\frac{\partial}{\partial \theta_{x i}} A_{i}\right] \mathrm{d} \theta_{x i}+\left[\frac{\partial}{\partial \theta_{y i}} A_{i}\right] \mathrm{d} \theta_{y i}+\left[\frac{\partial}{\partial \theta_{z i}} A_{i}\right] \mathrm{d} \theta_{z i}$

The $4 \times 4$ matrix $\left\{D_{i}\right\}_{\text {Transl } X}$ can be defined as follows:

Then $\delta A_{i}$ can be written in the form as Eq.(6).

$\delta A_{i}=\left\{D_{i}\right\}_{\text {Transl } X \mathrm{~d} X} X_{i}+\left\{D_{i}\right\}_{\text {Trans } Y \mathrm{~d} Y} Y_{i}+\left\{D_{i}\right\}_{\text {Trans } I Z \mathrm{~d} Z}$

$+\left\{D_{i}\right\}_{\mathrm{Rot} X \mathrm{~d} \theta_{x i}}+\left\{D_{i}\right\}_{\mathrm{Rot} Y \mathrm{~d} \theta_{y i}}+\left\{D_{i}\right\}_{\mathrm{Rot} Z \mathrm{~d} \theta_{z i}}$

Referring to the formula (4) (6), Eq.(7) can be attained as follows. 
$d_{i}=m_{1 i} \Delta X_{i}+m_{2 i} \Delta Y_{i}+m_{3 i} \Delta Z_{i}+m_{4 i} \Delta \theta_{\mathrm{x} i}+m_{5 i} \Delta \theta_{\mathrm{yi}}+m_{6 i} \Delta \theta_{z i}$

$\delta_{i}=m 7 i \Delta \theta_{x i}+m 8 i \Delta \theta_{y i}+m 9 i \Delta \theta_{z i}$

$m_{1 i}, m_{2 i}, m_{3 i}, m_{4 i}, m_{5 i}, m_{6 i}, m_{7 i}, m_{8 i}, m_{9 i}$ in Eq.(7) are all column vector, according to Eq.(7), the translational and rotational errors affect the position error of coordinate system, and the final azimuth error is only related with the rotation error.

\section{state space modeling in transmission process}

\section{Construction of transmission sequences and transmission process analysis}

The transfer relationship of transmission error is determined by machine tool transmission system. The transmission sequences are built according to the process from motor power source through gear transmission parts to final cutting tool. First the base coordinate system is set at machine tool bed, then the part coordinate systems $i$ are set at geometric center of each Transmission part, and the terminal cutting tool coordinate system is $N$.

\section{state space model of accumulated deviations in transmission process}

As shown in Eq.(8), the position and orientation deviation of the actual coordinate system relative to the ideal coordinate system is a vector consisting of six elements, which is micro amount relative to the size of parts.

$$
\widetilde{X}(i)=\left(\begin{array}{c}
d p_{i} \\
d \theta_{i}
\end{array}\right)=\left(\begin{array}{c}
d p_{i}^{x} \\
d p_{i}^{y} \\
d p_{i}^{z} \\
d \theta_{i}^{x} \\
d \theta_{i}^{y} \\
d \theta_{i}^{z}
\end{array}\right)
$$

The transmission errors transfer can be regarded as errors transfer in coordinate transformation . When transmitting to terminal cutting tool, the differential translation vector $d p_{N}$ and the differential rotation vector $d \theta_{N}$ of coordinates $\mathrm{N}$ relative to base coordinate system can be attained in Eq.(9).

$$
\left\{\begin{array}{c}
d p_{N}=\sum_{i=1}^{N}\left[R_{i-1} d p_{i}+p_{i-1} \times\left\{R_{i-1} d \theta_{i}\right\}\right] \\
d \theta_{N}=\sum_{i=1}^{N} R_{i-1} d \theta_{i}
\end{array}\right.
$$

$$
R_{i-1}, p_{i-1} \text { can be attained by Eq.(2) }
$$

When calculating the transmission errors accumulating to the $K$ transmission parts, the formula (9) can also be written as follows:

$$
\begin{aligned}
& \tilde{X}(\mathrm{~K})=\left[\begin{array}{l}
d p_{k} \\
d \theta_{\mathrm{k}}
\end{array}\right]=\left[\begin{array}{c}
W_{1} \\
0
\end{array}\right] \Delta X+\left[\begin{array}{c}
W_{2} \\
0
\end{array}\right] \Delta Y+\left[\begin{array}{c}
W_{3} \\
0
\end{array}\right] \Delta Z+\left[\begin{array}{c}
W_{4} \\
W_{5}
\end{array}\right] \Delta \theta_{x}+\left[\begin{array}{c}
W_{6} \\
W_{7}
\end{array}\right] \Delta \theta_{\mathrm{y}+}\left[\begin{array}{c}
W_{8} \\
W_{9}
\end{array}\right] \Delta \theta_{z} \\
& \Delta X=\left[\Delta X_{1}, \Delta X_{2}, \mathrm{~L}, \Delta X_{k}\right]^{T}, \mathrm{~L} \quad \Delta \theta_{z}=\left[\Delta \theta_{z 1}, \Delta \theta_{z 2}, \mathrm{~L}, \Delta \theta_{z k}\right]^{T} \text { are all } k \times 1 \text { column vectors shown in }
\end{aligned}
$$
Eq.(10), which can further be written as Eq.(11). 
$\left[\begin{array}{l}d p_{k}^{x} \\ d p_{k}^{y} \\ d p_{k}^{z} \\ d \theta_{k}^{x} \\ d \theta_{k}^{y} \\ d \theta_{k}^{z}\end{array}\right]=\left[\begin{array}{cccc}W_{(1,1), 1} & W_{(1,1), 2} & \cdot & W_{(1,1), k} \\ W_{(1,2), 1} & W_{(1,2), 2} & \cdot & W_{(1,2), k} \\ W_{(1,3), 1} & W_{(1,3), 2} & \cdot & W_{(1,3), k} \\ 0 & 0 & 0 & 0 \\ 0 & 0 & 0 & 0 \\ 0 & 0 & 0 & 0\end{array}\right]\left[\begin{array}{c}\Delta X_{1} \\ \Delta X_{2} \\ \cdot \\ \cdot \\ \cdot \\ \Delta X_{k}\end{array}\right]+\mathrm{L}+\left[\begin{array}{cccc}W_{8,1), 1} & W_{(8,1), 2} & \cdot & W_{(8,1), k} \\ W_{(8,2), 1} & W_{(8,2), 2} & \cdot & W_{(8,2), \mathrm{k}} \\ W_{(8,3), 1} & W_{(8,3), 2} & \cdot & W_{(8,3), \mathrm{k}} \\ W_{(9,1), 1} & W_{(9,1), 2} & \cdot & W_{(9,1), \mathrm{k}} \\ W_{9,2), 1} & W_{(9,2), 2} & \cdot & W_{(9,2), \mathrm{k}} \\ W_{(9,3), 1} & W_{(9,3), 2} & \cdot & W_{(9,3), \mathrm{k}}\end{array}\right]\left[\begin{array}{c}\Delta \theta_{z 1} \\ \Delta \theta_{z 2} \\ \cdot \\ \cdot \\ \cdot \\ \Delta \theta_{z k}\end{array}\right]$

$W_{i}=\left[\begin{array}{l}W_{(1,1), i} \\ W_{(1,2), i} \\ W_{(1,3), i}\end{array}\right]$ are all $3 \times 1$ column vectors shown in Eq.(11).

Eq.(12) can be derived combing Eq.(9) with Eq.(11) as follows:

$\left[\begin{array}{l}d p_{k}^{x} \\ d p_{k}^{y} \\ d p_{k}^{z} \\ d \theta_{k}^{x} \\ d \theta_{k}^{y} \\ d \theta_{k}^{z}\end{array}\right]=\left[\begin{array}{l}d p_{k-1}^{x} \\ d p_{k-1}^{y} \\ d p_{k-1}^{z} \\ d \theta_{k-1}^{x} \\ d \theta_{k-1}^{y} \\ d \theta_{k-1}^{z}\end{array}\right]+\left[\begin{array}{cccccc}W_{(1,1), \mathrm{k}} & W_{(2,1), \mathrm{k}} & W_{(3,1), \mathrm{k}} & W_{(4,1), \mathrm{k}} & W_{(6,1), \mathrm{k}} & W_{(8,1), \mathrm{k}} \\ W_{(1,2), \mathrm{k}} & W_{2,2), \mathrm{k}} & W_{(3,2), \mathrm{k}} & W_{(4,2), \mathrm{k}} & W_{(6,2), \mathrm{k}} & W_{(8,2), \mathrm{k}} \\ W_{(1,3), \mathrm{k}} & W_{(2,3), \mathrm{k}} & W_{(3,3), \mathrm{k}} & W_{(4,3), \mathrm{k}} & W_{(6,3), \mathrm{k}} & W_{(8,3), \mathrm{k}} \\ 0 & 0 & 0 & W_{(5,1), \mathrm{k}} & W_{(7,1), \mathrm{k}} & W_{(9,1), \mathrm{k}} \\ 0 & 0 & 0 & W_{(5,2), \mathrm{k}} & W_{(7,2), \mathrm{k}} & W_{9,2), \mathrm{k}} \\ 0 & 0 & 0 & W_{(5,3), \mathrm{k}} & W_{(7,3), \mathrm{k}} & W_{(9,3), \mathrm{k}}\end{array}\right] \times\left[\begin{array}{c}\Delta X_{k} \\ \Delta Y_{k} \\ \Delta Z_{k} \\ \Delta \theta_{x k} \\ \Delta \theta_{y k} \\ \Delta \theta_{z k}\end{array}\right]$

When transmitting to $K$ transmission parts ,the actual state $\widetilde{X}(\mathrm{k})$ is defined as a column vector consisting of six motion differential vectors, the transmission state model can be obtained from the state $\tilde{X}(k-1)$ to $\widetilde{X}(k)$ shown in Figure 5.

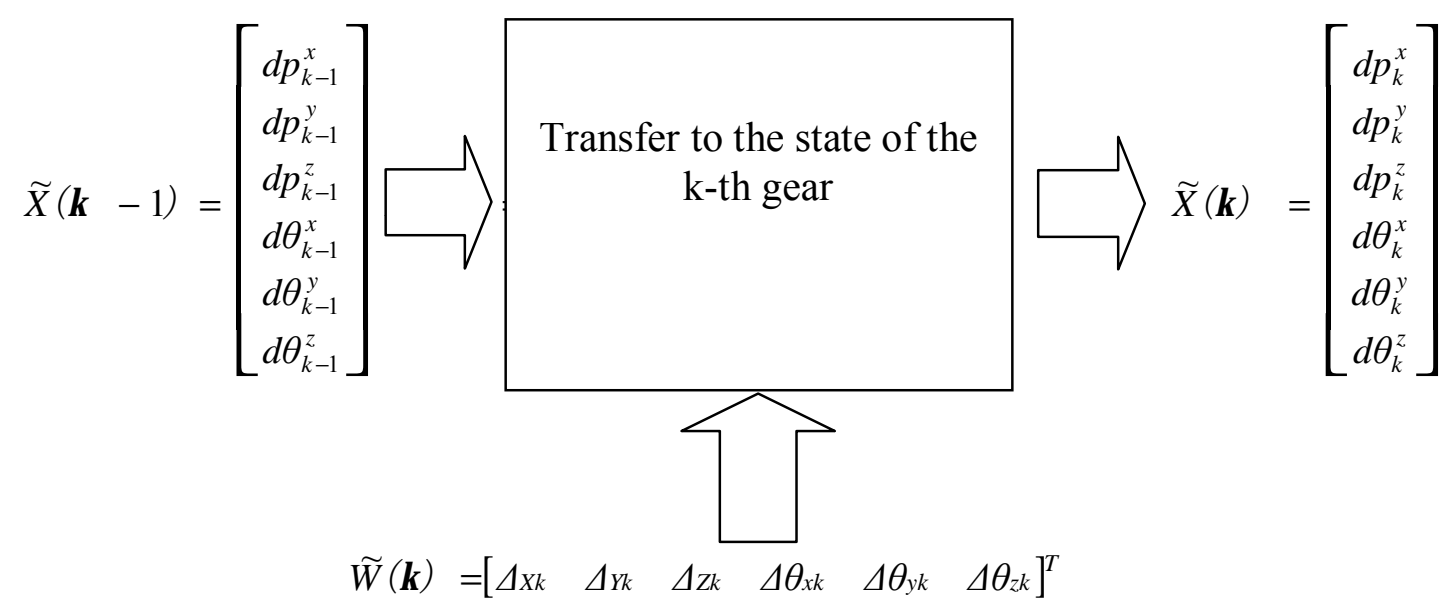

Figure 5. State space model

Definition: 
$A(\mathrm{k})=\mathrm{I}, \mathrm{F}(\mathrm{k})=\left[\begin{array}{cccccc}W_{(1,1), \mathrm{k}} & W_{(2,1), \mathrm{k}} & W_{(3,1), \mathrm{k}} & W_{(4,1), \mathrm{k}} & W_{(6,1), \mathrm{k}} & W_{(8,1), \mathrm{k}} \\ W_{(1,2), \mathrm{k}} & W_{2,2), \mathrm{k}} & W_{(3,2), \mathrm{k}} & W_{(4,2), \mathrm{k}} & W_{(6,2), \mathrm{k}} & W_{(8,2), \mathrm{k}} \\ W_{(1,3), \mathrm{k}} & W_{2,3), \mathrm{k}} & W_{(3,3), \mathrm{k}} & W_{(4,3), \mathrm{k}} & W_{6,3), \mathrm{k}} & W_{(8,3), \mathrm{k}} \\ 0 & 0 & 0 & W_{(5,1), \mathrm{k}} & W_{(7,1), \mathrm{k}} & W_{9,1), \mathrm{k}} \\ 0 & 0 & 0 & W_{5,2), \mathrm{k}} & W_{7,2), \mathrm{k}} & W_{9,2), \mathrm{k}} \\ 0 & 0 & 0 & W_{5,3), \mathrm{k}} & W_{(7,3), \mathrm{k}} & W_{(9,3), \mathrm{k}}\end{array}\right]$

Then the state space equation full form can be attained in Eq.(13).

$\left\{\begin{array}{c}\widetilde{X}(k)=A(k-1) \widetilde{X}(k-1)+F(k) \widetilde{W}(k)+\widetilde{g}(k) \\ \widetilde{Y}(k)=C(k) \widetilde{X}(k)+\widetilde{v}(k)\end{array}\right.$

Through the above analysis, the $\mathrm{CNC}$ hobbing spindle transmission system error model is attained based on state space equation, where the cumulative error vector $\widetilde{X}(\mathrm{k})$ is defined as k-th parts system state. The state transition matrix $A(k-1)$ is identity matrix, input matrix $F(k)$ shows the influence coefficient matrix. The error vector $\widetilde{W}(k)$ is obtained from $\mathrm{k}$-th parts design tolerances or experimental measurement.$Y(k)$ is the observation of k-th parts error, output matrix $C(k)$ is actually the selection matrix composed of 0 and 1 expressing our concern measurements. The mutual independent $\widetilde{g}(k)$ and $\widetilde{v}(k)$ indicates a system process noise and a measurement noise.

\section{Case analysis}

\section{Transmission process error calculation analysis}

According to the modeling method described in this paper, Taking YS3118 hobbing machine as an example, modeling and analyzing of the spindle transmission process, the error vectors can be attained by the transmission parts gear design tolerances shown in Table 1.

Table 1 each transmission parts error $(\mathrm{m} / \mathrm{s})$

\begin{tabular}{|c|c|c|c|c|c|c|}
\hline $\begin{array}{c}\text { Gear } \\
\text { number }\end{array}$ & $\begin{array}{c}\Delta x \\
\left(10^{-3} / \mathrm{m}\right)\end{array}$ & $\begin{array}{c}\Delta y \\
\left(10^{-3} / \mathrm{m}\right)\end{array}$ & $\begin{array}{c}\Delta z \\
\left(10^{-3} / \mathrm{m}\right)\end{array}$ & $\begin{array}{c}\Delta \theta_{x} \\
\left({ }^{\prime \prime}\right)\end{array}$ & $\begin{array}{c}\Delta \theta_{y} \\
(")\end{array}$ & $\begin{array}{c}\Delta \theta_{\mathrm{z}} \\
(\prime)\end{array}$ \\
\hline Gear 1 & 1.45 & 1.45 & 0.16 & 36 & 36 & 0 \\
\hline Gear 2 & 1.69 & 1.69 & 0.25 & 22 & 22 & 0 \\
\hline Gear 3 & 2.11 & 2.11 & 0.30 & 14 & 14 & 0 \\
\hline Gear 4 & 1.83 & 1.83 & 0.10 & 47 & 47 & 0 \\
\hline Gear 5 & 1.15 & 1.15 & 0.33 & 19 & 19 & 0 \\
\hline Gear 6 & 2.62 & 2.62 & 0.10 & 23 & 32 & 0 \\
\hline Gear 7 & 2.03 & 2.03 & 0.38 & 8 & 8 & 0 \\
\hline
\end{tabular}

According to the mathematical model, by taking the data in Table 1 into the above Eq.(11), the The six-dimensional error vector of the final hob coordinate system relative to the base coordinate system can be calculated as follows. 


$$
\widetilde{X}(N)=\left(\begin{array}{c}
d p_{N} \\
d \theta_{N}
\end{array}\right)=\left(\begin{array}{c}
651.1_{(\mathrm{mm})} \\
253.2_{(\mathrm{mm})} \\
1502.6_{(\mathrm{mm})} \\
0.236^{\circ} \\
0.509^{\circ} \\
0.378^{\circ}
\end{array}\right)
$$

$$
\tilde{X}(i)=\left(\begin{array}{c}
d p \mathrm{i} \\
d \theta \mathrm{i}
\end{array}\right)=\left(\begin{array}{c}
1.624_{\left(10^{-3} / \mathrm{mm}\right)} \\
0.226_{\left(10^{-3} / \mathrm{mm}\right)} \\
0.854_{\left(10^{-3} / \mathrm{mm}\right)} \\
0.236^{\circ} \\
0.509^{\circ} \\
0.378^{\circ}
\end{array}\right)
$$

The error vector above can be converted into that with respect to the ideal coordinate system:

The theoretical error vector above are calculated by the model, the data shows that $\Delta x>\Delta z>\Delta y, \Delta \theta_{y}>\Delta \theta_{z}>\Delta \theta_{x}$.

\section{experimental verification}

According to the gear hobbing machine processing principle, the error sensitivity directions are $\mathrm{X}$ and $\mathrm{Y}$ direction in hob coordinate system, so the error sources generating $\Delta \mathrm{X}$ and $\Delta \mathrm{Y}$ are sensitive error. $\Delta \mathrm{Z}$ and sensitive direction are perpendicular, so $\Delta \mathrm{Z}$ is the least sensitive direction. Therefore, the radial run-out deviation of hob shaft is only considered in this article while axial error is not .

First, let gear hobbing machine idle $0.5 \mathrm{~h}$ at the speed of $500 \mathrm{r} / \mathrm{min}$ to fully preheat to eliminate thermal errors. And then make the sensor head close to the hob shaft to get the radial run-out deviation value shown in figure 6. The transmission error curve takes hob rotating 360 degrees as a sampling period and the abscissa is hob rotational angle $\phi$.

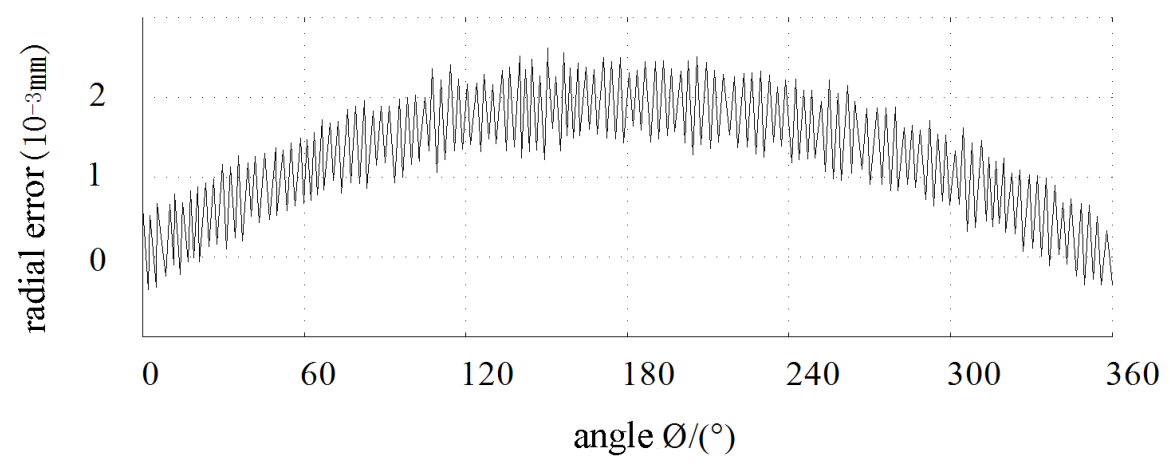

Figure 6. transmission error curve

The curve shows that the mean value of radial error is $1.333 \mathrm{um}$, which is close to $1.624 \mathrm{um}$ calculated by the model. Therefore, in order to verify the application scope of the model, spindle speed is set diversely and then the radial error is measured. Finally, the mean of radial run-out deviation of hob shaft in different rotating speed is obtained shown in figure 7.

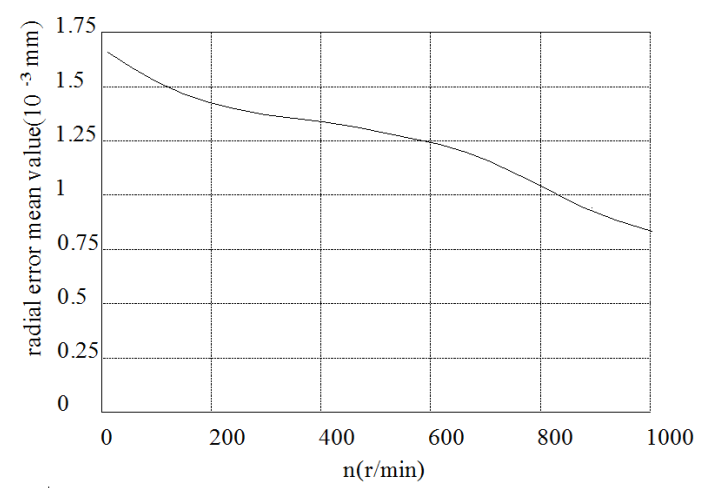

Figure 7. spindle radial error mean curve 
From the above analysis, when the speed of spindle is close to zero, the mean value of radial error is approaching to $1.66 \mathrm{um}$. Moreover, as the speed increases, the radial error gradually decreases. When it reaches maximum speed $1000 \mathrm{r} / \mathrm{min}$, its mean radial error is $0.823 \mathrm{um}$, whose difference is nearly $50 \%$ of the model calculated value. While the spindle runs in a low speed, the model calculated value is close to the actual measured value. Therefore, this model is suitable for low rpm spindle working conditions.

\section{Conclusion}

From the above analysis, the conclusion can be obtained that with the increasing spindle speed, the difference between calculated values and the actual measured value will be larger and the maximum error is almost approaching 50\%, so the model is only available in low rpm spindle conditions.

\section{Acknowledgments:}

This project is supported by National Natural Science Foundation of China "Research on coupling mechanism and control method for heterogeneous variation stream in complex manufacturing processes"(Grant No.51265024) and HongLiu Young Teachers Training Program of Lanzhou University of Technology.

\section{References}

[1]K.Y.Luo, S.M.Deng. Y3150E NC Hobbing Transformation and Application [J]. Machine Tool \& Hydraulics, 2004, 1: 144-145.

[2] J.L.Zhao,W.Guo,H.B.Yu. Evolution and Prospect of Research on Variation Transimission in Manufacturing Process [J].Chinese Mechanical Engineering, 2006, Supplement, 445-449.

[3] D.E.Whitney, O.L.Gilbert and M.Jastrzebski . Representation of Geometric Variations using Matrix Transforms for Statistical Tolerance Analysis in Assemblies[J]. Research in Engineering Design, 1994,6(4):191-210.

[4] R.Mantripragada , D .E. Whitney. The Datum Flow Chain: A Systematic Approach to Assembly Design and Modeling[J]. Research in Engineering Design, 1998,10(3):150-65.

[5] R.Mantripragada . Assembly-Oriented Design A Top-Down Approach to Modeling Assemblies[D]. Mech. Eng. Dept., Mass. Inst. Technol., Cambridge, 1997.

[6] R.Mantripragada, D .E.Whitney. Modeling and Controlling variation propagation in mechanical assemblies using state transition models[J].IEEE Transactions on Robotics and Automation, 1999,15(1):124-140.

[7] Z.Q.Tian, X.M.Lai, Z.X.Lin. State Space Model of Variations Stream Propagation In Mutilation Assembly Processes of Sheet Metal [J].Chinese Journal of Mechanical Engineering, 2007,43(2):202-209.

[8] J.Hong, J.K.Guo, Z.G.Liu, X.P.Wu.Assembly Accuracy Prediction and Adjustment Process Modeling of Precision Machine Tool Based on State Space Model [J].Chinese Journal of Mechanical Engineering, 2013,49(6):114-121.

[9] T.S.Wang. Robot kinematics and dynamics [M]. Xi'an University of Electronic Science and Technology Press, 1990. 